\title{
Multi-focal Myxopapillary Ependymoma in the Lumbar and Sacral Regions Requiring Cranio-spinal Radiation Therapy: A Case Report
}

\author{
Hirotaka Andoh', Yoshiharu Kawaguchi', Shoji Seki', Yumiko Asanuma', \\ Jun Fukuoka², Shin Ishizawa ${ }^{2}$, Tomoatsu Kimura ${ }^{1}$ \\ 'Department of Orthopaedic Surgery, University of Toyama Faculty of Medicine, Toyama, Japan \\ ${ }^{2}$ Department of Pathology, University of Toyama Faculty of Medicine, Toyama, Japan
}

Ependymomas are uncommon tumors that arise in the brain, spinal cord or cauda equina. Myxopapillary ependymomas is located exclusively in the conus medullaris or cauda equina, or film terminale region. In most myxopapillary ependymomas, the histological examination reveals low mitotic activity that is associated with a low MIB-1 labeling index (LI). The prognosis is generally favorable, when the appropriate treatment, including a total resection, is performed. The authors encountered a 39-year-old man with multifocal type of myxopapillary ependymomas compressing the cauda equina from the L2 to L3 level and L5-S1 level. A subtotal resection of the tumor was carried out. The histological examination revealed extremely high mitotic activity with a MIB-1 LI of 9.1\%. Therefore, cranio-spinal radiation was added after surgery. The postoperative course was uneventful over the 3.5 year follow-up period.

Key Words: Myxopapillary ependymoma, Lumbar and sacral regions, Cranio-spinal radiation therapy, MIB-1 index, Tumor recurrence

\section{Introduction}

Ependymomas are relatively rare tumors that arise in the brain, spinal cord or cauda equina. Spinal ependymomas are less frequent than intracranial ependymomas. The World Health Organization (WHO) classified ependymomas into the following four categories: subependymomas (WHO grade 1), myxopapillary ependymomas (WHO grade 1), ependymomas (WHO grade 2) and anaplastic ependymomas (WHO grade 3) [1]. Among these types, a myxopapillary ependymoma is characterized as a slow growing tumor with preferential manifestation in young adults. Myxopapillary ependymoma is located exclusively in the conus medullaris or the cauda equina, or film terminale region, and is rarely found as a multi-focal type. In most myxopapillary ependymomas, the histological examination reveals low mitotic activity that is associated with a low MIB-1 labeling index (LI) [2]. The prognosis is generally favorable [3]. However, late recurrence, distant metastases and subarachnoid disseminations have been reported [4-6]. We report an unusual case of multi-focal myxopapillary ependymoma with extremely high mitotic activity that arose in the cauda equine. In this case, the mitotic activity of the tumor was extremely high compared to previously reported cases [2]. A surgical resection and cranio-spinal radiation were performed as treatments.

Received Jan 11, 2010; 1st Revised Feb 5, 2010; 2nd Revised Feb 12, 2010; Accepted Feb 12, 2010

Corresponding author: Yoshiharu Kawaguchi, MD, PhD

Department of Orthopaedic Surgery, University of Toyama Faculty of Medicine

2630 Sugitani, Toyama 930-0194, Japan

Tel: +81-76-434-7353, Fax: +81-76-434-5035, E-mail: zenji@med.u-toyama.ac.jp 


\section{Case Report}

A 39-year-old man presented with gradually increasing bilateral buttock pain. He showed intermittent claudication after walking $500 \mathrm{~m}$. The physical examination revealed cauda equina syndrome below the L2 level. He had dysuria. Dejerine signs, postural factor and a positive Kemp sign. The straight leg raising test was also positive in the bilateral lower extremities. The deep tendon reflexes in the lower extremities were diminished, but the reflexes in the upper extremities were normal. Motor palsy was not evident and no sensory disturbance was observed in the upper or lower extremities. The plain radiographs did not reveal any abnormalities. Lumbar magnetic resonance imaging (MRI) demonstrated an intradural tumor compressing the cauda equina from the L2 to L3 level and L5-S1 level (Fig. 1). The tumor did not extend beyond the spinal canal (Fig. 2). The tumor showed low intensity compared to the spinal cord on the T1-weighted image and high intensity on the T2-weighted image. Gadolinium enhancement was observed in the tumor. The tumor mass was not detected in the cranial, cervical or thoracic levels by MRI.

An en-bloc lumbar laminectomy was carried out and a total resection of the tumor was performed with an opening of the dura mater. The tumor showed a round and redishgray colored mass covered with a thin membrane. The tumor was not attached to the dura mater or cauda eqina at the L2-3 levels. As the tumor was firmly attached to the S2 spinal nerve, it had to be removed piece by piece under a microscope at the L5-S1 level. After removing the tumor, the dura mater could be sutured completely. No dural cyst, due to the leakage of spinal fluid, remained after surgery.

The gross size of the tumor from the L2-3 level was $4 \times$ $1.3 \mathrm{~cm}$. Macroscopically, the tumor was soft with a glossy grayish color and focal hemorrhagic appearance. Histologically, the tumor from the L2-3 and L5-S1 levels showed various morphologies with extensive myxoid degeneration. Most of the tumor cells contained eosinophilic rich cytoplasm with bipolar processes. The major part of the tumor showed reticular arrangement with numerous hyalinized vessels distributed evenly. The radial arrangement of the cellular processes against the vascular wall was obvious (Fig. 3A). Some parts showed marked mucoid degeneration surrounded by layers of tumor cells (Fig. 3B). The cellular atypia was not conspicuous and the area of necrosis could not be identified. Immunohistochemical analysis revealed the tumor cells to be positive for glial fibrillary acidic protein (GFAP) and S-100, and negative for keratin, CAM5.2 and epithelial membrane antigen (EMA) (Fig. 3C). A diagnosis of myxopapillary ependymoma was made based on the histological appearance, immunohistochemical profile and clinical presentation. According to WHO classification, all myxopapillary ependymoma falls into grade 1 . However, the current case showed relatively frequent mitosis of up to 2/10 hpf and a high MIB-1 LI of 9.1\%, which raised the possibility of a more aggressive tumor (Fig. 3D).

Cranio-spinal radiation therapy was performed 1 month after surgery. The amount of radiation was $30 \mathrm{~Gy}$ for the

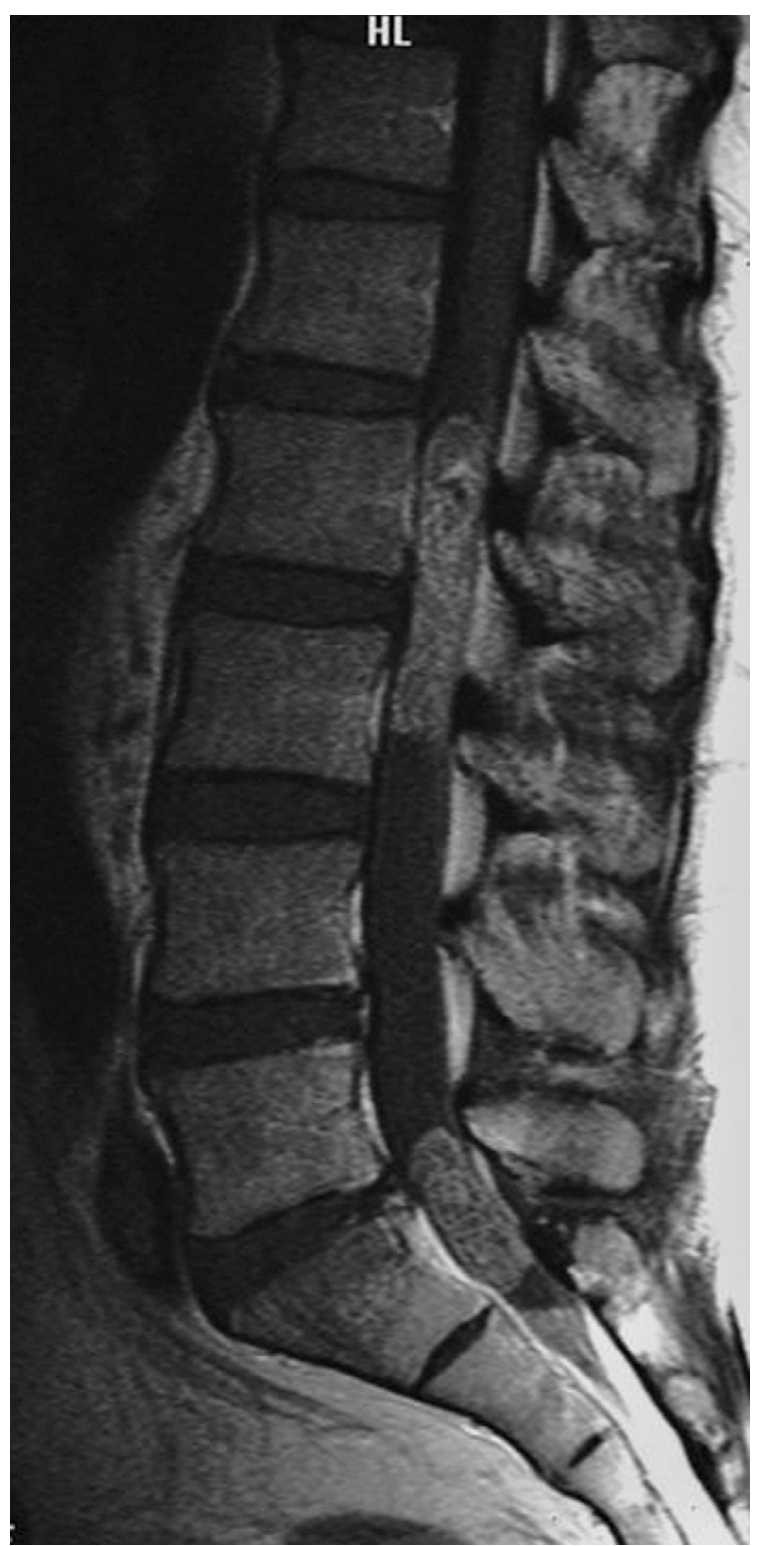

Fig. 1. Enhanced magnetic resonance imaging sagittal view demonstrating an intradural tumor compressing the cauda equina from the L2 to L3 level and L5-S1 level. 

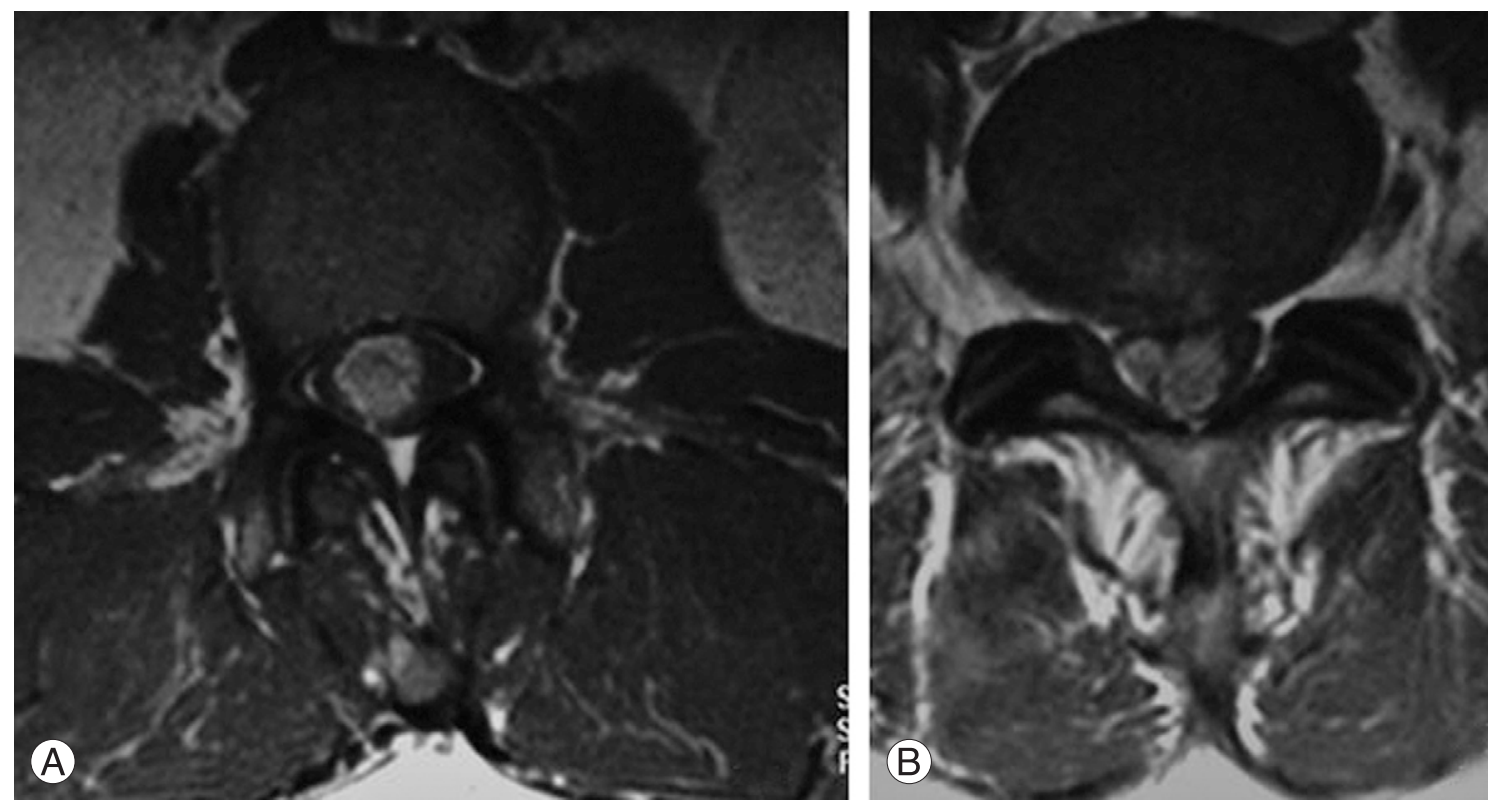

Fig. 2. Enhanced magnetic resonance imaging axial view showing the intra canal tumor at the L2 level (A) and S1 level (B).
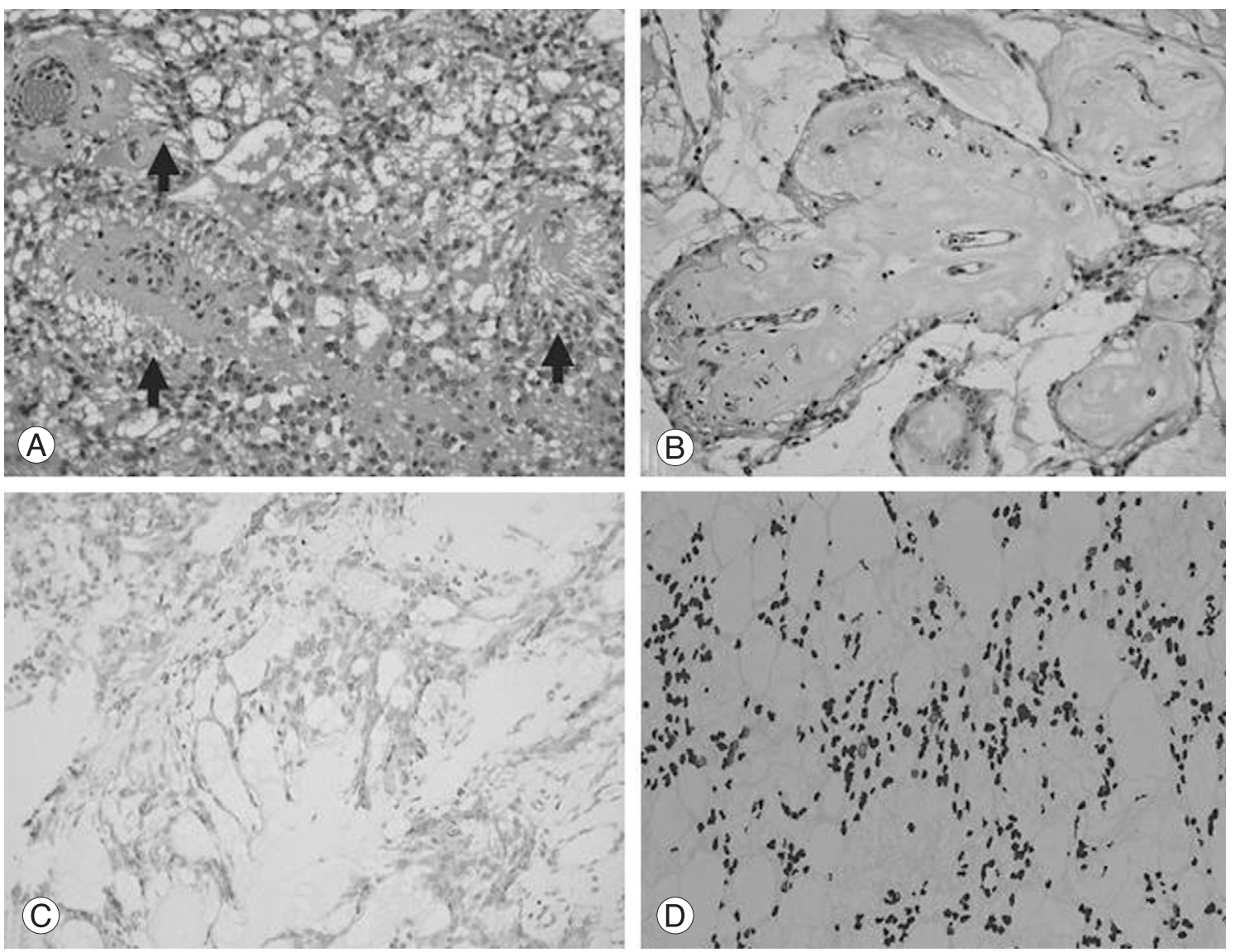

Fig. 3. Pathology of the tumor. (A) Tumor cells show radial processes around the vessels (arrows). (B) Areas with marked mucoid degeneration. Note the mucoid materials surrounding the vessels. (C) Tumor cells are diffusely positive for glial fibrillary acidic protein. (D) The tumor shows high a MIB-1 labeling index of $9.1 \%$. 


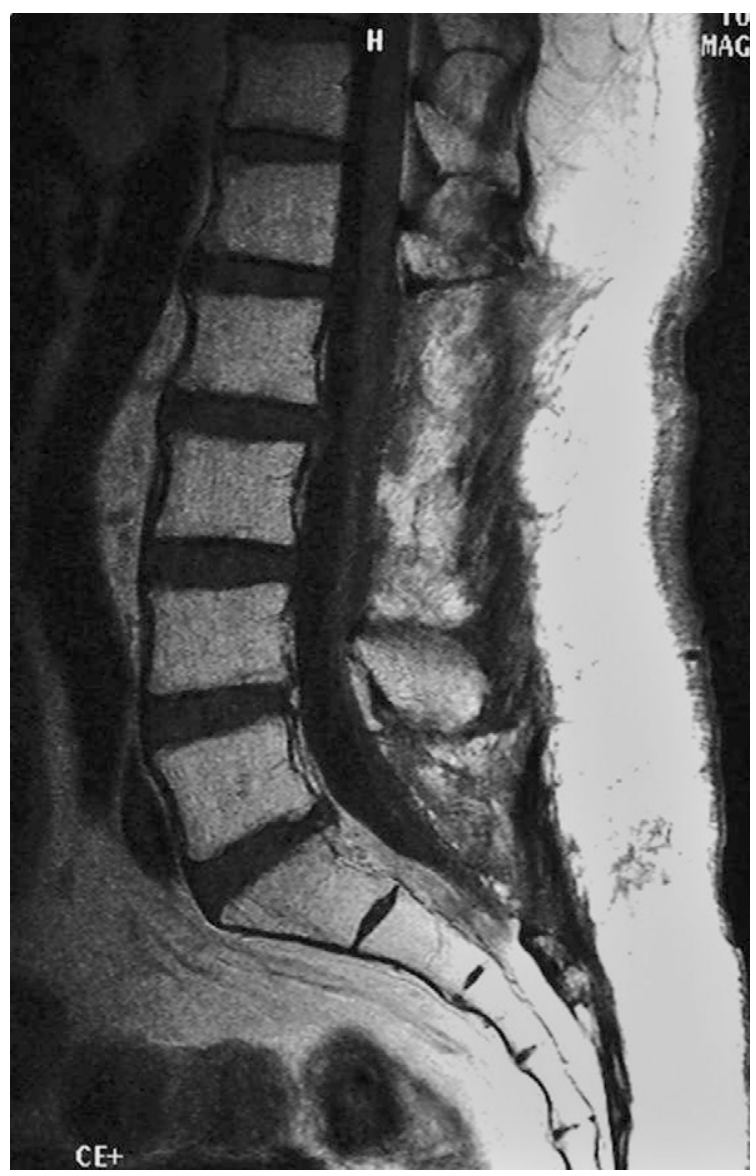

Fig. 4. Enhanced magnetic resonance imaging sagittal view of the lumbar spine 3.5 year after surgery showing no tumor recurrence.

brain-C2, C3-T10 and T11-S levels, and $10 \mathrm{~Gy}$ for the L1-S levels. Symptoms, such as nausea, vomiting, a disorder of the sense of taste and depilation, as well as leukocytopenia $(<2,000 / \mu \mathrm{l})$ were observed after radiation therapy. However, the patient recovered in 2 months and returned to his sedentary work. He has been followed for 3.5 years since the operation and radiation therapy. He remains asymptomatic and the MRI of his brain, spinal cord and cauda equina have not showed any recurrence (Fig. 4).

\section{Discussion}

It was reported that myxopapillary ependymoma is located exclusively in the conus medullaris and cauda equina region [1]. In the present case, the tumor was the multifocal type at the L2-3 and L5-S1 levels. The multifocal type of myxopapillary ependymoma is relatively rare. Sonneland et al. [3] reported that both the film terminale and conus medullaris were involved in $35 \%$ of patients. Hanbali et al.
[7] reported that 2 out of 26 patients had multifocal tumors. Myxopapillary ependymoma is encapsulated in most cases and a total resection can be performed safely in the majority of patients. However, a total resection is sometimes difficult in cases with involvement of the cauda equina or the film terminale. In the present case, a subtotal resection for the tumor was performed at the L5-S1 region because the tumor was firmly attached to the S2 spinal nerve.

Approximately $90 \%$ of spinal cord ependymomas have a benign pathology and are reported to be slow growing $[1,3]$. A favorable prognosis is expected when a total resection is performed. However, a 50-70\% recurrence rate of spinal ependymoma has been reported after a subtotal resection [46]. In addition, subarachnoid dissemination and external metastasis has been observed occasionally $[5,6,8]$. Therefore, it is necessary to pay close attention for possible tumor recurrence in cases with a subtotal resection. In recent years, a variety of cell proliferation markers have been used to predict tumor recurrence and/or metastasis. The MIB-1 antibody was shown to be a reliable marker of cell proliferation. The MIB-1 antibody reacts with a nuclear antigen expressed during the active phases of the cell cycle, including G1, G2, S and M phases. MIB-1 LI has been used as an operational marker of cell proliferation in a variety of tumors. In myxopapillary ependymoma, Prayson [2] analyzed MIB-1 LI in 14 patients. His data suggested that the MIB LI level was $0.9 \%$ on average, ranging from 0 to $5.5 \%$ and MIB-1 IL was high in 2 cases with tumor recurrence. Although he reported that MIB-1 LI is an unreliable predictor of tumor recurrence, it should be noted that the patients with a higher MIB-1 LI had tumor recurrence. Compared to his data, the MIB-1 LI in the present case was extremely high $(9.1 \%)$, indicating a high likelihood of tumor growth, recurrence and/or metastasis.

In the present case, cranio-spinal radiation therapy was carried out after surgery because the patient had a multifocal type of myxopapillary ependymoma, high MIB-1 LI and the tumor was removed in stages. In the series reported by Hanbali et al. [7], one case of the multi-focal type had tumor recurrence after surgery. Sonneland et al. [3], in their analysis of 77 cases with myxopapillary ependymoma, reported that total removal had a recurrence rate of $10 \%$, whereas those that were removed either piecemeal or subtotally had recurrence rates of $19 \%$. Therefore, adjuvant radiation therapy was recommended for cases without complete resection [9]. Although there was no evidence of a doseresponse relationship between the amount of radiation and 
tumor progression, most institutes recommend radiation doses in the range of 40-50 Gy. A total of $100 \mathrm{~Gy}$ radiation was applied from the brain to the spinal cord and the cauda equina. Each of the lesions received $30 \mathrm{~Gy}$. In this case, it was necessary to discontinue the radiation therapy due to the unfavorable side effects, such as nausea, vomiting, disorder of sense of taste and depilation, and leukocytopenia after radiation. Radiation myelopathy, which generally occurs at doses $>55$ Gy during the follow-up, should also be considered.

The postoperative course of the present case was uneventful during the follow-up. The patient had no tumor recurrence in the brain, spinal cord or the cauda equina. There have been some cases of tumor recurrence, particularly in cases with a subtotal resection [9]. Regarding chemotherapy, one case report described the use of Imatinib for recurrent spinal ependymoma [10]. Chemotherapy was not added because there is little data available on the benefit of chemotherapy for spinal cord ependymomas. However, it will be necessary to continue following this patient very carefully.

In conclusion, we encountered a case of multi-focal spinal ependymoma with high mitotic activity. A subtotal resection was performed and cranio-spinal radiation was added. The patient had an uneventful clinical course over the 3.5 year follow-up period.

\section{REFERENCES}

1. Louis DN, Ohgaki H, Wiestler OD, et al. The 2007 WHO classification of tumours of the central nervous system. Acta Neuropathol 2007;114:97-109.

2. Prayson RA. Myxopapillary ependymomas: a clinicopathologic study of 14 cases including MIB-1 and p53 immunoreactivity. Mod Pathol 1997;10:304-10.

3. Sonneland PR, Scheithauer BW, Onofrio BM. Myxopapillary ependymoma: a clinicopathologic and immunocytochemical study of 77 cases. Cancer 1985;56:883-93.

4. Guidetti B, Mercuri S, Vagnozzi R. Long-term results of the surgical treatment of 129 intramedullary spinal gliomas. J Neurosurg 1981;54:323-30.

5. Cooper PR. Outcome after operative treatment of intramedullary spinal cord tumors in adults: intermediate and long-term results in 51 patients. Neurosurgery 1989;25:855-9.

6. Gomez DR, Missett BT, Wara WM, et al. High failure rate in spinal ependymomas with long-term follow-up. Neuro Oncol 2005;7:254-9.

7. Hanbali F, Fourney DR, Marmor E, et al. Spinal cord ependymoma: radical surgical resection and outcome. Neurosurgery 2002;51:1162-72.

8. Graf M, Blaeker H, Otto HF. Extraneural metastasizing ependymoma of the spinal cord. Pathol Oncol Res 1999;5:56-60.

9. Akyurek S, Chang EL, Yu TK, et al. Spinal myxopapillary ependymoma outcomes in patients treated with surgery and radiotherapy at M.D. Anderson Cancer Center. J Neurooncol 2006;80:177-83.

10. Fakhrai N, Neophytou P, Dieckmann K, et al. Recurrent spinal ependymoma showing partial remission under Imatimib. Acta Neurochir (Wien) 2004;146:1255-8. 